
\title{
$\begin{array}{ll}\text { Research Square } & \begin{array}{l}\text { Preprints are preliminary reports that have not undergone peer review. } \\ \text { They should not be considered conclusive, used to inform clinical practice, } \\ \text { or referenced by the media as validated information. }\end{array}\end{array}$
}

\section{Effect of a Calcium-Enriched Lemon Beverage on Bone Metabolism and Bone Mineral Density in Postmenopausal Women: A Double-Blind, 11-Month, Intervention Study}

\author{
Hiromi lkeda \\ Prefectural University of Hiroshima \\ Tadayuki lida ( $\nabla$ iida@pu-hiroshima.ac.jp) \\ https://orcid.org/0000-0003-4934-3576 \\ Masanori Hiramitsu \\ Frontier Laboratories for Value Creations, SAPPORO HOLDINGS LTD. \\ Takashi Inoue \\ Foodtechnology Laboratories for Value Creation, SAPPORO HOLDINGS LTD. \\ Satomi Aoi \\ Hiroshima Kenritsu Daigaku Gakujutsu Joho Center Toshokan \\ Miho Kanazashi \\ Prefectural University of Hiroshima \\ Fumiko Ishizaki \\ Professor Emeritus of Prefectural University of Hiroshima \\ Toshihide Harada \\ Prefectural University of Hiroshima
}

Research article

Keywords: Calcium-Supplemented Lemon Beverages, Bone Metabolism, Bone Mineral Density, Postmenopausal Women

Posted Date: February 12th, 2020

DOI: https://doi.org/10.21203/rs.2.23049/v1

License: (9) (7) This work is licensed under a Creative Commons Attribution 4.0 International License. Read Full License 


\section{Abstract}

Background

Key approaches to preventing the onset of osteoporosis are to maximize peak bone mass (PBM) in early adulthood and to minimize the inevitable loss of bone mineral density (BMD) after menopause. Consequently, osteoporosis attracts a great deal of social as well as medical attention in Japan, a country experiencing population aging.

Methods

The present randomized, controlled study aimed to elucidate how bone metabolism and bone mineral density (BMD) are affected by the consumption of a lemon juice-containing, calcium-enriched beverage. The effectiveness of this investigational product was evaluated in 79 postmenopausal women. The subjects were assigned to three groups. The first two groups received calcium-supplemented (LECA group) and unsupplemented lemon (LE group) beverages, respectively. The third group (control) received no intervention. The LECA and LE groups consumed one bottle (290 ml) of the appropriate investigational product every day for 11 consecutive months.

Results

The results showed that the regular consumption of this lemon beverage promoted Ca absorption through the chelating function of citric acid, attenuating bone resorption. The results also suggest that the suppression of bone resorption inhibits bone formation mediated by the differentiation and activity of osteoblasts and leads to the prevention of high-turnover bone metabolism.

Conclusion

This hypothesis is further supported by the novel finding that the regular consumption of the lemon beverage increases the BMD of both the lumbar spine and femur. We believe that the Ca-supplemented lemon beverage used in the present study will be instrumental in developing effective strategies for the prevention of osteoporosis.

\section{Background}

Key approaches to preventing the onset of osteoporosis are to maximize peak bone mass (PBM) in early adulthood and to minimize the inevitable loss of bone mineral density (BMD) after menopause [1-3]. Low estrogen levels associated with menopause lead to insufficient suppression of bone resorption. This increases bone resorption and consequently decreases bone mass, resulting in postmenopausal osteoporosis [4]. In postmenopausal women, the turnover rate of bone metabolism is accelerated, leading to a rapid progression of bone loss [5]. Hence, the incidence of osteoporosis is exceptionally high in these women. They also have a higher risk of fractures, a serious complication of osteoporosis. In the elderly, calcium (Ca) balance becomes negative due to an age-related decrease in $\mathrm{Ca}$ absorption in the intestine. A negative $\mathrm{Ca}$ balance also results from vitamin $\mathrm{D}$ deficiency, which is caused, for example, by a reduction in dietary intake or by a decrease in biosynthesis owing to a lack of ultraviolet light exposure. An impairment in vitamin D activation in the kidney is also associated with a decrease in $\mathrm{Ca}$ absorption, leading to a negative $\mathrm{Ca}$ balance. Low levels of $\mathrm{Ca}$, in turn, induce mild hyperparathyroidism and increase the concentration of parathyroid hormone. Subsequently, this hormone stimulates osteoclast activity and releases Ca from bone, resulting in the normal level of Ca in the blood [6]. These observations indicate that the attenuation of high-turnover bone metabolism is vital for the prevention of postmenopausal osteoporosis.

Patients with osteoporosis have few subjective symptoms. However, once the disease advances and fractures occur, these patients experience a variety of difficulties. Osteoporotic fractures are even associated with a higher risk of mortality [7]. Consequently, osteoporosis attracts a great deal of social as well as medical attention in Japan, a country experiencing population aging. Drugs for treating osteoporosis are prescribed after a definitive diagnosis has been made, and most of these drugs simply aim to inhibit a reduction in BMD. Thus, preventing bone loss is far more important than treating established osteoporosis.

A wide range of osteoporosis studies performed to date indicate that, from the perspective of preventive medicine, lifestyle (especially dietary and exercise habits) is crucial in preventing the onset of this disease [8-10]. Recently, certain research efforts have focused on the functions of nutrients (such as dietary supplements) and investigated their effects on BMD and bone metabolism. Yamamura et al. [11] reported an increase in BMD in healthy adult women who had received a six-month intervention with milk basic protein (MBP). Similarly, Aoe et al. [12,13] demonstrated that, in healthy pre- and early postmenopausal women, an intervention with MBP increased BMD in 6-8 months by inhibiting bone resorption. In these studies, BMD was assessed only at the forearm, calcaneus, and lumbar spine, although the measurement was performed using dual energy X-ray absorptiometry (DEXA), the current standard modality for osteoporosis diagnosis and fracture risk prediction. For osteoporotic fracture risk assessment, the International Society for Clinical Densitometry recommends the use of the trunk bones (including the spine and hip) for the DEXA-based evaluation of BMD [14]. Thus, to increase the accuracy of the assessment, BMD should be determined at the lumbar spine and femur [15]. As for the duration of intervention, many clinical studies using therapeutic drugs for osteoporosis set it to one year, and they successfully detect increases in BMD after this treatment period [16, 17]

Previously, our group examined the effectiveness of a Ca-supplemented lemon beverage for the prevention of osteoporosis in postmenopausal women who had regularly consumed the beverage for five months [18].

The effects of this beverage were evaluated by measurement of the trunk bones, including the spine and hip, and bone metabolism markers using a doubleblind study design. As a result, the beverage was able to suppress accelerated bone metabolism and preserve BMD of the lumbar spine compared with the control group. We hypothesized that these effects were due to the chelating activity of citric acid contained in the beverage. However, there was no significant 
difference in the BMD of the proximal femur. Bone turnover of the lumbar spine, which is approximately $60 \%$ cancellous bone, is active, but the femoral neck, which is approximately $25 \%$ cancellous bone, has a high ratio of cortical bone [19,20]. Thus, a long examination period is necessary to assess the effects on the femur BMD.

Therefore, we extended the intervention period of this study and investigated the effectiveness of a Ca-supplemented lemon beverage, which was consumed every day for 11 consecutive months, in addition to managing product intake records.

\section{Methods \\ Subjects}

Study subjects were healthy postmenopausal middle-aged and elderly women. They were recruited as volunteers through public advertising in $\mathrm{M}$ city. The exclusion criteria were as follows: (1) individuals with serious comorbid diseases, such as myocardial or cerebral infarction and hepatic or renal dysfunction; (2) individuals who treats warfarin medicine; and (3) any individuals unsuitable for this study, as judged by the principal investigator. Study participants were provided with full details regarding the study (such as aims and methods) at an orientation session offered prior to enrollment in the study. Written, informed consent was obtained from 100 participants. Of these, 79 were selected for statistical analysis, since they had undergone all scheduled examinations. In the present research, these examinations were conducted at the beginning of the study (prior to intervention with an investigational product), as well as five and 11 months after study initiation. This study used a randomized, controlled design. For anonymization, each subject was assigned an identification code. Subjects were divided by researchers (T. lida and $\mathrm{H}$. I) into three groups matched for age and body mass index (BMI) (Age: \pm 3 age $\left.\square B M I \otimes \pm 3 \mathrm{~kg} / \mathrm{m}^{2}\right)$. In the LECA group ( 28 women), subjects received a lemon beverage supplemented with $\mathrm{Ca}$. In the LE group ( 28 women), subjects received an unsupplemented lemon beverage. The third group (control, 23 women) received no intervention. Three groups were divided by researchers (M. H). Subjects allocated to the intervention group and data analysts घresearcher $\square$ T.lida and H.l[were kept blinded to the allocation. In the present study, subjects in the control group received no placebo products and led their normal lives. For each subject in the LECA and LE groups, the appropriate investigational product was provided using a double-blind procedure. This study was conducted in accordance with the Declaration of Helsinki and was approved by the Ethics Committee of the Prefectural University of Hiroshima (Approval Number: 15MH025-01). This study is registered at UMIN Clinical Trials Registry, number UMIN000018952.

\section{Clinical Examinations and Experimental Design}

The study period was between December 2015 and December 2016. Each subject in the LECA and LE groups consumed one serving (290 ml) of the appropriate investigational product every day for 11 months. Subject characteristics, BMD, and bone metabolism markers were evaluated at study initiation (pre-intervention) and five and 11 months after the beginning of the intervention at the Prefectural University of Hiroshima, Mihara campus

\section{Subject Characteristics}

Age and the number of years since menopause were obtained by interview. Physical measurements evaluated were height, weight, and BMI, at Prefectural University of Hiroshima, Mihara Campus.

2. BMD

Using the X-ray bone densitometer Discovery (Toyo Medic, Tokyo, Japan), BMDs (g/cm2) of the lumbar spine (L2-L4) and left proximal femur were determined $[21,22]$. During the study period, the densitometer was calibrated 99 times. The calibration results ( $\mathrm{mean}=1.038 \mathrm{~g} / \mathrm{cm} 2, \mathrm{SD}=0.003 \mathrm{~g} / \mathrm{cm} 2$, and $\mathrm{CV}=0.37 \%$ ) were all within the normal ranges specified by the manufacturer (Hologic, Inc., Marlborough, MA, USA).

\section{Bone Metabolism Markers}

The following markers were evaluated: bone resorption markers, serum tartrate-resistant acid phosphatase type 5b (TRACP-5b) and urinary type I collagen cross-linked N-telopeptide (u-NTx); bone formation markers, bone alkaline phosphatase (BAP) and osteocalcin (OC); and bone matrix-related marker, undercarboxylated osteocalcin (ucOC).

To obtain serum for assaying TRACP-5b, BAP, OC, and ucOC, blood samples collected from study subjects were centrifuged at 3,000 rpm for 7 min. For assaying u-NTx, urine samples were used without pretreatment. All assays were performed by Fukuyama Medical Laboratory Co., Ltd. (Hiroshima, Japan) using the following commercially available kits: Osteolinks "TRAP-5b" (Nittobo Medical Co., Ltd., Tokyo, Japan) for TRACP-5b; Access Ostase (Beckman Coulter Co., Ltd., Brea, CA, USA) for BAP; BGP IRMA "LSI M" (LSI Medience Co., Ltd., Tokyo, Japan) for OC; Picolumi ucOC (EIDIA Co., Ltd., Tokyo, Japan) for ucOC; and Osteomark (Alere Medical Co., Ltd., Tokyo, Japan) for u-NTx.

4. Compositions of the Investigational Products and Experimental Protocol

The investigational products were provided by the collaborating research institution Pokka Sapporo Food \& Beverage LTD. The amounts of principal ingredients in these products are shown in Table 1. Study subjects were simply instructed to consume one bottle (290 ml) of the investigational product per day. There were no instructions as to what time of the day the product should be taken. Subjects were also instructed to keep product intake records, and these records were reviewed every three months. The mean adherence rate for subjects selected for statistical analysis was $94.2 \%$. The amount of lemon juice in both the unsupplemented and Ca-supplemented investigational products was $30 \mathrm{ml} /$ bottle. The amount of Ca in the Ca-supplemented investigational product was $350 \mathrm{mg} /$ bottle. 
Table 1. Compositions of Test Beverages

\begin{tabular}{|llll|}
\hline & & LE$^{1}$ Group & LECA $^{2}$ Group \\
\hline Energy & $(\mathrm{kcal} / 100 \mathrm{ml})$ & 7 & 17 \\
\hline Protein & $(\mathrm{g} / 100 \mathrm{ml})$ & 0 & 0 \\
\hline Fat & $(\mathrm{g} / 100 \mathrm{ml})$ & 0 & 0 \\
\hline Carbohydrate & $(\mathrm{g} / 100 \mathrm{ml})$ & 1.8 & 4.4 \\
\hline Ash & $(\mathrm{g} / 100 \mathrm{ml})$ & 0 & 0.3 \\
\hline Sodium & $(\mathrm{mg} / 100 \mathrm{ml})$ & 0 & 1.4 \\
\hline Lemon juice & $(\mathrm{ml} / 100 \mathrm{ml})$ & 10.4 & 10.4 \\
\hline Calcium & $(\mathrm{mg} / 100 \mathrm{ml})$ & 0 & 120.7 \\
\hline
\end{tabular}

${ }^{1}$ They received an unsupplemented lemon beverage.

2 They received a lemon beverage supplemented with Ca.

5. Data Analysis

The normality of data was assessed using the Shapiro-Wilk test. For baseline clinical variables, values are expressed as means with standard deviation in parentheses when the assumption of normality was met. For those that did not meet the normality assumption, data are presented as medians with interquartile range (25-75th percentiles) in parentheses. After 11 months of intervention, the effects of investigational products and intervention duration on clinical variables (BMD and bone metabolism marker levels) were examined. When the normality assumption of clinical variable data was satisfied, two-way analysis of variance, after adjusting for age and BMI, was performed. In this analysis, one of the two factors was the three subject groups that received either an investigational product (LECA and LE) or no product (control). The other factor was the duration of intervention [pre-intervention (at study initiation), five months of intervention, and 11 months of intervention]. The dependent variable was one of the clinical variables. When the normality assumption was not met, Friedman's test was performed for each subject group. In addition to these analyses, a net gain in each clinical variable was calculated for each subject by subtracting the baseline value from the value obtained after 11 months of intervention. The net gain was then divided by the baseline value to determine the ratio of change from baseline. When the normality and equal variance assumptions of these ratios were both satisfied, they were analyzed by one-way analysis of variance, followed by the Dunnett multiple comparison test for each subject group. When the normality assumption was met but the assumption of equal variance was violated, the ratios were assessed by the Welch test. When both assumptions were violated, the Kruskal-Wallis rank-sum test was used. Both the Welch and Kruskal-Wallis tests were followed by the Holm multiple comparison test. The level of significance for all statistical tests was set at $p<$ 0.05 .

\section{Results}

\section{Subject Characteristics and Clinical Variables at Baseline}

Baseline subject characteristics and the baseline values of clinical variables are shown in Tables 2 and 3 , respectively. There were no significant differences in any of the subject characteristics among the three (LECA, LE, and control) groups. 
Table 2

Physical characteristics of study subjects

\begin{tabular}{|c|c|c|c|c|c|c|c|c|c|c|c|c|c|c|c|c|c|c|}
\hline \multirow[b]{3}{*}{$\begin{array}{l}\text { Clinical } \\
\text { Variable }\end{array}$} & \multicolumn{15}{|c|}{ Subject Group } & \multicolumn{3}{|c|}{ Total $(n=79)$} \\
\hline & \multicolumn{5}{|c|}{ Control $(n=23)$} & \multicolumn{5}{|c|}{$\operatorname{LE}^{1}(n=28)$} & \multicolumn{5}{|c|}{$\operatorname{LECA}^{2}(n=28)$} & \multirow[b]{2}{*}{ Mean } & \multirow[b]{2}{*}{ (SD) } & \multirow[b]{2}{*}{ N } \\
\hline & Mean & $\left(S D^{4}\right)$ & $\operatorname{Min}^{5}$ & - & $\operatorname{Max}^{6}$ & Mean & (SD) & Min & - & Max & Mean & (SD) & Min & - & Max & & & \\
\hline Age (y) & 63.9 & (7.1) & 48.0 & - & 80.0 & 64.9 & (5.8) & 56.0 & - & 81.0 & 65.2 & $(5.2)$ & 57.0 & - & 82.0 & 64.7 & $(6.0)$ & $4 i$ \\
\hline $\begin{array}{l}\text { Years after } \\
\text { menopause } \\
\text { (y) }\end{array}$ & 13.0 & $(6.9)$ & 0.0 & - & 29.0 & 15.1 & $(9.2)$ & 5.0 & - & 51.0 & 16.4 & (7.6) & 2.0 & - & 31.0 & 14.9 & $(8.1)$ & 0. \\
\hline Height (cm) & 154.3 & (5.4) & 141.1 & - & 162.8 & 154.5 & $(4.6)$ & 147.5 & - & 162.5 & 152.8 & (5.6) & 143.2 & - & 164.1 & 153.9 & $(5.2)$ & 1 \\
\hline $\begin{array}{l}\text { Baseline } \\
\text { Weight (kg) }\end{array}$ & 50.8 & $(10.1)$ & 30.3 & - & 79.0 & 53.4 & (8.5) & 41.9 & - & 72.9 & 52.3 & $(7.2)$ & 40.3 & - & 65.7 & 52.3 & (8.5) & 31 \\
\hline $\begin{array}{l}\text { Baseline } \\
\mathrm{BMI}^{3} \\
\left(\mathrm{~kg} / \mathrm{m}^{2}\right)\end{array}$ & 21.6 & (3.5) & 16.6 & - & 32.5 & 22.2 & (3.5) & 17.4 & - & 31.6 & 22.4 & $(2.8)$ & 17.8 & - & 29.0 & 22.1 & (3.2) & 11 \\
\hline \multicolumn{19}{|c|}{ There were no significant differences in any of these variables among the three subject groups. } \\
\hline \multicolumn{19}{|c|}{${ }^{1}$ They received an unsupplemented lemon beverage. } \\
\hline \multicolumn{19}{|c|}{2 They received a lemon beverage supplemented with Ca. } \\
\hline \multicolumn{19}{|c|}{${ }^{3}$ body mass index } \\
\hline \multicolumn{19}{|c|}{${ }^{4}$ standard deviation } \\
\hline \multicolumn{19}{|c|}{${ }^{5}$ minimum value } \\
\hline${ }^{6}$ maximal v & & & & & & & & & & & & & & & & & & \\
\hline
\end{tabular}


Table 3

Measurements before the intervention for the study subjects

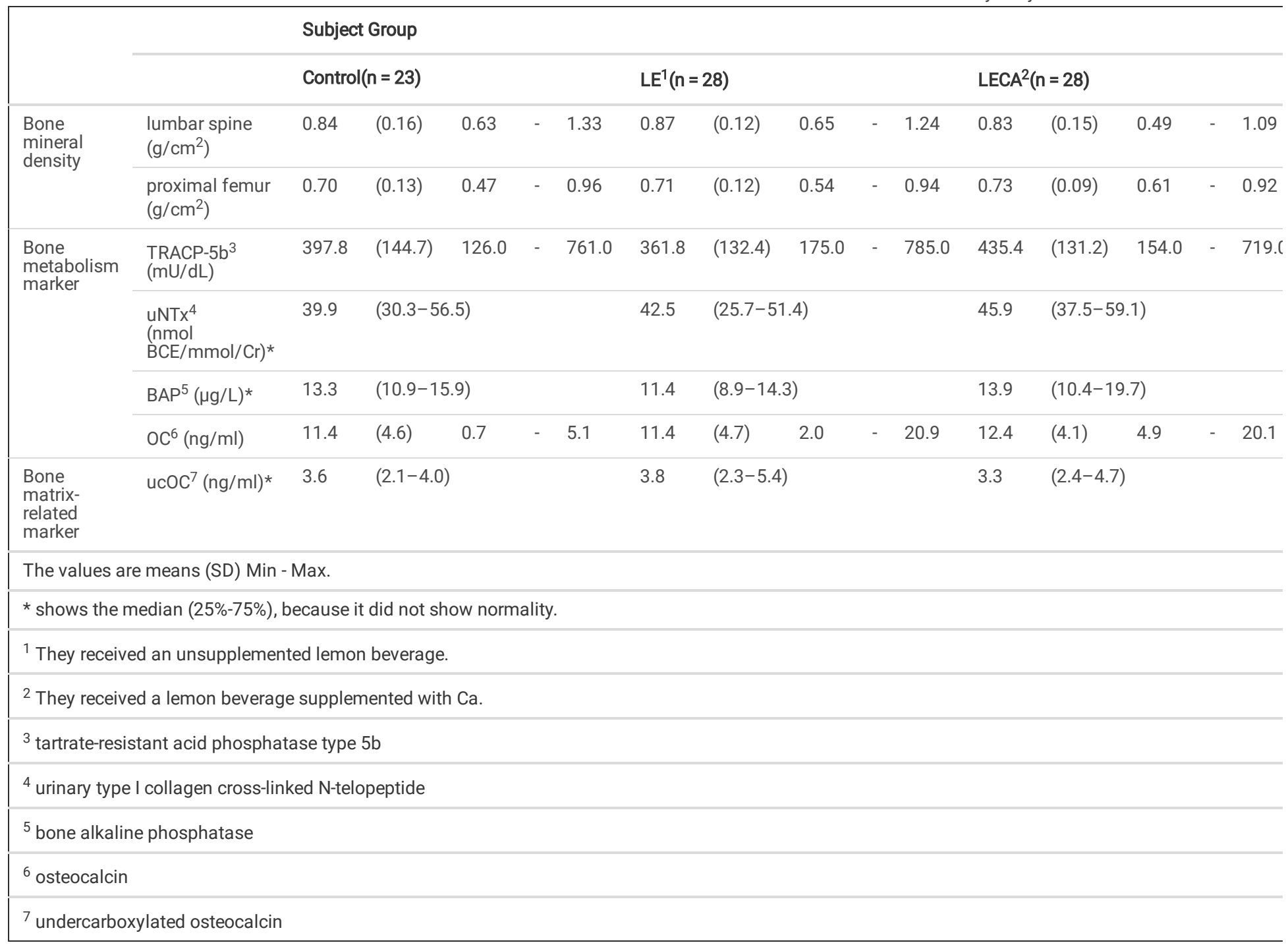

\section{Changes in BMD}

There were no significant differences in the mean BMD of the lumbar spine among the three subject groups $(p=0.666)$ or different time points $(p=0.956)$. However, there was a significant interaction effect $(p<0.001)$ (Fig. 1-a). When the ratios of change from baseline were calculated for lumbar spine BMD after 11 months of intervention, a positive value was obtained for the LECA group (0.03), while negative values were obtained for the LE and control groups (LE:-0.00,control:-0.04). The differences in these values between the LECA and control groups and between the LE and control groups were both significant ( $p$ $<0.001$ ) (Table 4). 
Table 4

Ratio of changes after 11 months of intervention

\begin{tabular}{|c|c|c|c|c|c|c|c|c|c|c|c|c|c|c|c|c|c|}
\hline \multicolumn{2}{|c|}{ Ratio of changes } & \multicolumn{13}{|c|}{ Subject Group } & \multicolumn{3}{|c|}{ p-value } \\
\hline & & \multicolumn{3}{|c|}{ Control( $(n=23)$} & \multicolumn{5}{|c|}{$\operatorname{LE}^{1}(n=28)$} & \multicolumn{5}{|c|}{$\operatorname{LECA}^{2}(n=28)$} & \multicolumn{3}{|c|}{ vs LE } \\
\hline \multirow[t]{2}{*}{$\begin{array}{l}\text { Bone } \\
\text { mineral } \\
\text { density }\end{array}$} & $\begin{array}{l}\text { lumbar spine } \\
\left(\mathrm{g} / \mathrm{cm}^{2}\right)\end{array}$ & -0.04 & $(0.03)$ & -0.09 & - & 0.03 & 0.00 & $(0.02)$ & -0.04 & - & 0.04 & 0.03 & $(0.03)$ & -0.01 & - & 0.11 & $\nabla 0.001^{\mathrm{a}}$ \\
\hline & $\begin{array}{l}\text { proximal femur } \\
\left(\mathrm{g} / \mathrm{cm}^{2}\right)\end{array}$ & -0.02 & $(0.02)$ & -0.05 & - & 0.05 & -0.02 & $(0.02)$ & -0.05 & - & 0.02 & 0.00 & $(0.01)$ & -0.05 & - & 0.03 & $0.995^{\mathrm{a}}$ \\
\hline \multirow[t]{4}{*}{$\begin{array}{l}\text { Bone } \\
\text { metabolism } \\
\text { marker }\end{array}$} & $\begin{array}{l}\text { TRACP-5b3 } \\
(\mathrm{mU} / \mathrm{dL})\end{array}$ & 0.03 & $(0.27)$ & -0.32 & - & 0.69 & 0.08 & $(0.17)$ & -0.25 & - & 0.49 & -0.10 & $(0.16)$ & -0.39 & - & 0.22 & $0.685^{\mathrm{a}}$ \\
\hline & $\begin{array}{l}\mathrm{uNTT}^{4} \\
(\mathrm{nmol} \\
\mathrm{BCE} / \mathrm{mmol} / \mathrm{Cr})^{*}\end{array}$ & 0.00 & $(-0.45-$ & $0.30)$ & & & -0.01 & $(-0.29-$ & $0.22)$ & & & -0.16 & $(-0.38$ & .03) & & & .000 \\
\hline & $\operatorname{BAP}^{5}(\mu \mathrm{g} / \mathrm{L})^{*}$ & 0.17 & $(0.02)$ & -0.15 & - & 0.54 & 0.26 & $(0.17)$ & 0.01 & - & 0.83 & 0.03 & $(0.15)$ & -0.29 & - & 0.37 & $0.135^{\mathrm{a}}$ \\
\hline & $\mathrm{OC}^{6}(\mathrm{ng} / \mathrm{ml})$ & 0.00 & $(-0.29-$ & $0.21)$ & & & -0.02 & $(-0.12-$ & $0.15)$ & & & -0.27 & $(-0.36$ & $0.10)$ & & & .590 \\
\hline $\begin{array}{l}\text { Bone } \\
\text { matrix- } \\
\text { related } \\
\text { marker }\end{array}$ & $\mathrm{ucOC}^{7}(\mathrm{ng} / \mathrm{ml})^{*}$ & -0.10 & $(-0.33-$ & $0.12)$ & & & -0.14 & $(-0.25-$ & $0.12)$ & & & -0.22 & $(-0.43$ & $0.03)$ & & & .580 \\
\hline \multicolumn{18}{|c|}{ The values are means (SD) Min - Max. } \\
\hline \multicolumn{18}{|c|}{ * shows the median (25\%-75\%), because it did not show normality. } \\
\hline \multicolumn{18}{|c|}{ a shows the results obtained by the Dunnett multiple comparison test after analyzed by one-way analysis of variance. } \\
\hline \multicolumn{18}{|c|}{${ }^{b}$ shows the results obtained by the Kruskal- Wallis test. } \\
\hline \multicolumn{18}{|c|}{${ }^{\mathrm{c}}$ shows the results obtained by $\mathrm{p}$ value adjustment method (holm) after the Kruskal- Wallis test. } \\
\hline \multicolumn{18}{|c|}{${ }^{1}$ They received an unsupplemented lemon beverage. } \\
\hline \multicolumn{18}{|c|}{${ }^{2}$ They received a lemon beverage supplemented with Ca. } \\
\hline \multicolumn{18}{|c|}{${ }^{3}$ tartrate-resistant acid phosphatase type $5 \mathrm{~b}$} \\
\hline \multicolumn{18}{|c|}{${ }^{4}$ urinary type I collagen cross-linked N-telopeptide } \\
\hline \multicolumn{18}{|c|}{${ }^{5}$ bone alkaline phosphatase } \\
\hline \multicolumn{18}{|l|}{${ }^{6}$ osteocalcin } \\
\hline 7 undercarbc & ated osteocalcin & & & & & & & & & & & & & & & & \\
\hline
\end{tabular}

Similarly, there were no significant differences in the mean BMD of the femur among the three subject groups $(p=0.771)$ or among different time points $(p=$ 0.413). Again, the interaction effect was significant $(p<0.001)$ (Fig. 1-b). The ratios of change from baseline calculated for femoral BMD after 11 months of intervention were positive for the LECA group (0.00), but negative for the LE and control groups (LE:-0.02,control:-0.02). The difference in these ratios between the LECA and control groups was significant (pष0.001) (Table 4).

\section{Changes in the Concentrations of Bone Metabolism Markers}

Mean TRACP-5b concentrations did not show any significant differences among the three subject groups $(p=0.840)$ or different time points $(p=0.967)$. However, the interaction effect was significant $(p<0.001)$ (Fig. $2-a)$. When the ratios of change from baseline were calculated for TRACP-5b levels after 11 months of intervention, a negative value was obtained for the LECA group (-0.10), whereas positive values were obtained for the LE and control groups (LE:0.08,control:0.03). The difference in these values between the LECA and control groups was significant $(p=0.002)(T a b l e 4)$. In the case of $u-N T x$, its concentrations did not differ significantly over the course of the study period in all three subject groups (Fig. 2-b). Similarly, there were no significant differences in the ratios of change from baseline after the 11-month intervention (Table 4).

As for the mean concentrations of OC, there were no significant differences among the three subject groups $(p=0.788)$ or different time points $(p=0.254)$. The interaction effect was significant $(p=0.006)$ (Fig. 2-c). When the ratios of change from baseline were determined for $0 \mathrm{C}$ levels after 11 months of intervention, the value obtained for the LECA group $(-0.27)$ was significantly $(p<0.001)$ smaller than that for the LE group $(-0.02)$ (Table 4$)$. In the case of BAP

concentrations, they showed significant $(p<0.001)$ differences during the study period in all three subject groups (Fig. 2-d). The ratios of change from baseline 
calculated for BAP concentrations after 11 months of intervention were positive for all three subject groups (LECA:0.03, LE:0.26, control:0.17). However, the LECA group showed a significantly $(p=0.007)$ smaller value than the control group (Table 4).

Finally, ucOC concentrations in the LECA group showed significant $(p<0.001)$ differences during the study period (Fig. 3). However, there were no significant differences in the ratios of change from baseline for ucOC levels after the 11-month intervention (Table 4).

\section{Discussion}

Menopausal and postmenopausal women have low levels of the female hormone estrogen, leading to an increased risk of osteoporosis [23-25]. Preservation of BMD through the attenuation of high-turnover bone metabolism is crucial in preventing postmenopausal osteoporosis. However, in menopausal and postmenopausal women, the lack of estrogen negatively affects the maintenance of BMD. Despite this, the present study succeeded in demonstrating the favorable effects of a Ca-supplemented lemon beverage on BMD and bone metabolism in healthy postmenopausal middle-aged and elderly women (mean age $\pm S D, 64.7 \pm 6.0$ years).

Both the Ca-supplemented and unsupplemented investigational products consumed by the LECA and LE groups, respectively, contained 30 ml of lemon juice per bottle. In addition, each bottle of the Ca-supplemented investigational product contained $350 \mathrm{mg}$ of Ca. For adult women, the recommended dietary reference intake for Ca is approximately $700 \mathrm{mg}$ per day [26]. In the present study, one-half of this value (i.e., $350 \mathrm{mg}$ ) was used as the daily intake amount. In determining the amount of lemon juice in the investigational products, the health survey conducted by Domoto et al. was used [27]. They found that, in a lemon-growing region of Hiroshima Prefecture, individuals with high daily lemon consumption (approximately 30 ml, on average) had significantly lower blood pressure than those with low consumption. Based on this observation, the lemon juice amount was set to $30 \mathrm{ml}$. The amounts of Ca and lemon juice in the investigational products are within the ranges of quantities one normally obtains by consuming ordinary foods. There have been no specific reports indicating that these amounts of Ca or lemon juice pose potential health hazards. Thus, they were deemed safe.

In the subjects of this study (postmenopausal women), there was a significant interaction effect for the concentrations of the bone resorption marker TRACP$5 b$. When the ratios of change from baseline were calculated for TRACP-5b levels after 11 months of intervention, negative and positive values were obtained for the LECA and control groups, respectively, and the difference between these two values was significant. A significant interaction effect was also detected for the concentrations of the bone formation marker OC. As for BAP concentrations, the ratios of change from baseline calculated after 11 months of intervention were positive for all three subject groups. However, the values obtained for the LECA and control groups were significantly different, as a substantial increase was observed in the control group. In a study using a calcium supplement, Smith et al. [28] previously demonstrated that Ca supplementation was effective in preventing bone loss in middle-aged women. Similarly, Reid et al. [29] showed that Ca supplementation could reduce the rate of loss of total body BMD in postmenopausal women. In these investigations, subjects received 1,000-1,500 mg of Ca per day. Separately, in an MBP intake study with 84 young women, Zou et al. [30] demonstrated that the concentrations of the bone resorption marker serum NTx were significantly lower in women who had consumed MBP for eight months. Moreover, in a study with 33 healthy adult women, Aoe et al. [12] showed that the concentrations of u-NTx and urinary deoxypyridinoline were significantly decreased in women who had consumed MBP for six months. The same research group subsequently conducted a similar study with 32 early postmenopausal women and demonstrated that u-NTx levels were significantly reduced by six-month intake of MBP [13]. However, despite these significant reductions in bone resorption marker levels, no changes in the concentrations of bone formation markers were detected in the above MBP intake studies. A decrease in bone formation takes place following the inhibition of bone resorption. This coupling phenomenon likely causes a delay in the reduction of bone formation markers after the concentrations of bone resorption markers decrease [22]. In an intervention study using an antiosteoporotic agent [31], no significant changes were detected in $\Sigma G S / D$ after six months of treatment. In contrast, similar treatment approaches had favorable clinical effects (such as a decrease in the loss of BMD and an increase in BMD) after 1-2 years of intervention [16, 17, 32, 33]. Thus, an extended period (at least one year) of intervention would be required to verify the effects of osteoporosis prevention strategies. Both changes in bone resorption and bone formation markers could be observed in this study.

We postulate that the reason changes in both bone resorption and formation markers were detected is attributable to the chelating function of citric acid (which is mainly responsible for the acidic flavor of lemons). Lemon juice is a rich source of citric acid (1.44 g/oz) [34]. Previous studies [35-37] have demonstrated that this organic acid acts as a chelating agent and promotes the absorption of minerals (such as Ca and iron) by increasing their solubility in water. We hypothesize that, in the present study, this property of citric acid accounts for the reductions in the concentrations of bone resorption and bone formation markers in the LECA group. In the present research, each subject in the LECA group received 350 mg of Ca daily through the consumption of the Casupplemented lemon beverage. This amount was lower than the levels of Ca supplementation used in the studies described above [28, 29]. However, when this lemon beverage was consumed regularly, the chelating activity of citric acid likely facilitated Ca absorption in the subjects and maintained their blood Ca levels within the normal range. This presumably eliminated the need for supplying Ca from bone and suppressed osteoclast-mediated bone resorption, resulting in the inhibition of osteoblast function and the attenuation of bone formation. This hypothesis is consistent with the results of our previous study in which we evaluated the effects of a Ca-supplemented lemon beverage in postmenopausal women [38].

In this previous study, the duration of intervention was short (six months). Therefore, we were unable to clearly determine whether the observed effects of the lemon beverage were caused by a component(s) of lemon juice or by an increase in Ca absorption achieved by the chelating function of citric acid in lemons. To this end, we conducted the present study with an extended period (11 months) of intervention using three subject groups (control, LE, and LECA) and succeeded in demonstrating the effects of the chelating function of citric acid. Based on this result, we believe that the effects of citric acid's chelating activity have profound implications for the prevention of osteoporosis. We further found that the concentration of the bone matrix-related marker ucOC was significantly reduced in the LECA group after 11 months of intervention. This suggests that, in bone tissue, vitamin $\mathrm{K}$ is involved in both the inhibition of bone resorption and the promotion of bone formation. ucOC, which is produced in the absence of vitamin $\mathrm{K}$ in the blood and represents the uncarboxylated form, is unable to bind $\mathrm{Ca}$ [39]. Thus, high ucOC levels indicate the low vitamin $\mathrm{K}$ status of bone, i.e., the conditions where bone formation and bone resorption are 
inhibited and promoted, respectively. Vitamin K promotes bone formation by stimulating osteoblast differentiation and increasing the levels of certain proteins involved in bone formation (such as BAP) [35]. Furthermore, it prevents bone resorption by decreasing osteoclast differentiation and inhibiting osteoblast apoptosis [40]. A study that investigated the age-related changes of ucOC [41] found that the concentrations of this protein increased with age in middle-aged and elderly women. Separately, in postmenopausal women, the turnover rate of bone metabolism is known to increase in an age-dependent manner [9]. These observations suggest that vitamin $\mathrm{K}$ is insufficient in bone when both bone formation and bone resorption are accelerated (i.e., the state of high-turnover bone metabolism). This would decrease the incorporation of carboxylated OC into the bone matrix and stimulate the release of ucOC into the circulation, elevating its serum levels. In the present study, the concentrations of both bone resorption and bone formation markers decreased in the LECA group after 11 months of intervention. The aim of this study was not to elucidate the changes in ucOC levels during bone turnover and metabolism. However, based on the present results, we can still speculate that, in bone tissue, vitamin $\mathrm{K}$ functions as both an inhibitor of bone resorption and a stimulator of bone formation. Two concepts that emerged from our research (i.e., the Ca-supplemented lemon beverage tested in this study attenuates high-turnover bone metabolism, and vitamin $\mathrm{K}$ suppresses bone resorption and promotes bone formation) are novel and potentially have broad implications.

The interaction effect was significant for the mean BMD of the lumbar spine and the proximal femur. The ratio of change from baseline was calculated, and the differences in these values between the LECA and control and LE groups were significant for lumbar spine BMD after 11 months of intervention. Moreover, there were significant differences in these values between the LECA and control groups for proximal femur BMD. Bone is constantly renewed through a process called remodeling [42]. This process elegantly regulates the balance between osteoclastic and osteoblastic activities, thereby preserving dynamic bone homeostasis. In other words, normal bone tissue is maintained through the resorption of an appropriate amount of old bone and the subsequent formation of new bone [43]. Changes in bone metabolism are induced during menopause; bone resorption increases and this drives bone formation, resulting in high bone turnover. However, resorption ratios are much higher than formation ratios, causing a reduction in bone mass [44, 45]. In the present study, normal levels of blood Ca likely inhibited bone resorption. This would have suppressed the differentiation and activity of osteoblasts and caused the attenuation of high-turnover bone metabolism. Fractures caused by osteoporosis often occur, for example, in the vertebral body, proximal femur, lower leg bones, and distal radius. However, the most common type of osteoporotic fracture is a vertebral body fracture [22]. In addition, proximal femoral fractures are associated with a decrease in activities of daily living and an increase in the risk of becoming bedridden [46]. Most bones in the body are composed of cortical bone and trabecular bone. Therefore, the effects of osteoporosis prevention strategies must be assessed using both the lumbar spine and femur, which consist mainly of trabecular bone and cortical bone, respectively [47]. In the present study, BMD was examined in these two sites [i.e., the lumbar spine (L2-L4) and proximal femur], and the LECA group had a significantly higher density at both sites than the control group based on the ratio of change from baseline. The finding that the Ca-supplemented lemon beverage increased bone density at these two sites is highly significant and strongly suggests the effectiveness of this approach to osteoporosis prevention.

The physical measurements of each subject in the present study were similar to the mean values obtained from an age-matched Japanese population [48]. This suggests the external validity of the present findings to the general population of postmenopausal women, at least in the context of their physical characteristics. One of the limitations of the present research is that the study subjects were not hospitalized patients or institutionalized individuals. They were volunteers interested in health studies. Thus, the present subjects could have been biased towards healthy people or health-conscious individuals. Another limitation is that a dietary survey was not conducted for each subject. Furthermore, the possibility that the subjects had diverse lifestyles differing in, for example, daily habits, cannot be excluded. We will address these issues in our future studies. Despite these limitations, unprecedented results were obtained from this clinical research, which was conducted with subjects who consumed a Ca-supplemented investigational product, while otherwise leading their normal lives. In our previous study [18], in which the effects of the same investigational products were assessed after five months of intervention, we proposed a hypothesis that citric acid in lemons increases the absorption of dietary Ca. The results obtained here (after 11 months of intervention) are similar to those found in the previous study, thus supporting this hypothesis.

\section{Conclusions}

A clinical study with an extended period (11 months) of intervention was performed to examine the effects of a Ca-supplemented lemon beverage on the prevention of osteoporosis. The results showed that, in postmenopausal women, the regular consumption of this lemon beverage promoted Ca absorption through the chelating function of citric acid, causing the attenuation of bone resorption. The results also suggest that the suppression of bone resorption inhibits bone formation mediated by the differentiation and activity of osteoblasts and leads to the prevention of high-turnover bone metabolism. This hypothesis is further supported by the novel finding that the regular consumption of the lemon beverage increases the BMD of both the lumbar spine and proximal femur. We believe that the Ca-supplemented lemon beverage used in the present study will be instrumental in developing effective strategies for the prevention of osteoporosis.

\section{Abbreviations}

\section{BAP}

bone alkaline phosphatase

BMD

bone mineral density

BMI

body mass index

$\mathrm{Ca}$

calcium

DEXA 
dual energy X-ray absorptiometry

MBP

milk basic protein

OC

osteocalcin

PBM

peak bone mass

TRACP-5b

tartrate-resistant acid phosphatase type $5 \mathrm{~b}$

ucOC

undercarboxylated osteocalcin

u-NTx

urinary type I collagen cross-linked $\mathrm{N}$-telopeptide

\section{Declarations}

\section{Acknowledgements}

The authors are grateful to the volunteer of the M city which participated in this study. We also thank Prof. H. Miyaguchi of Hiroshima University and Prof. Y. Nitta of Hiro-shima Shudo University for experiment assistance with this study.

\section{Author information}

Affiliations

Department of Health and Welfare, Prefectural University of Hiroshima, 1-1 Gakuen-cho, Mihara, Hiroshima, 723-0053, Japan

Hiromi Ikeda, Tadayuki lida, Satomi Aoi, Miho Kanazashi, Toshihide Harada

Frontier Laboratories for Value Creation, SAPPORO HOLDINGS LTD., 10 Okatome, Yaizu, Shizuoka 425-0013, Japan

Masanori Hiramitsu

Foodtechnology Laboratories for Value Creation, SAPPORO HOLDINGS LTD., 1189-4 Nippa-cho Kohoku-ku, Yokohama 223-0057, Japan

Takashi Inoue

Professor Emeritus of Prefectural University of Hiroshima, 1-1 Gakuen-cho, Mihara, Hiroshima, 723-0053, Japan

Fumiko Ishizaki

Corresponding author

Correspondence to Tadayuki lida.

Additional information

Competing interests: All other authors have no conflict of interest.

Author Contributions: Conceptualization, T. lida., M.H., T. Inoue and T.H.; Methodology, T. lida. and M.H.; Validation, T. lida., M.H. and T.H.; Formal Analysis, H.I., and T. lida.; Investigation, T. lida., H.I., S.A., M.K. and F.I.; Resources, M.H.; Data Curation, H.I.; Writing - Original Draft Preparation, H.I.; Writing - Review \& Editing, T. lida., M.H., and T.H.; Visualization, H.I.; Supervision, T. lida.; Project Administration, T. lida.; Funding Acquisition, T. lida. All authors have read and approved the manuscript.

Funding: The present study was supported by SAPPORO HOLDINGS LTD. M.H. and T. Inoue are employees of SAPPORO HOLDINGS LTD., the funders of this study, and they had a role in the design of the study and discussed the data.

\section{Availability of data and materials}

Data cannot be shared publicly because datasets have ethical or legal restrictions for public deposition owing to inclusion of sensitive information from the human participants. Based on regulations for ethical guidelines in Japan, the ethical review board of the Faculty of Health and Welfare, Prefectural University of Hiroshima imposed restrictions on the data collected in this study. Obtained data and material were used only for the present study and are available only to the researchers who participated in the study project.

Consent for publication $\bowtie$ Not Applicable.

\section{References}


1. Bachrach LK. Acquisition of optimal bone mass in childhood and adolescence. Trends Endocrinol. Metab. 2001, 12: 22-8.

2. Orimo S, Kuroda T, Onoe Y, Sato Y, Ohta H. Age-related distribution of bone and skeletal parameters in 1,322 Japanese young women. J. Bone Miner. Metab. 2009, 27: 698-704.

3. Southard R.N, Morris J.D, Mahan J.D, Hayes J.R, Torch M.A, Sommer A, Zipf W.B. Bone mass in healthy children: measurement with quantitative DXA. Radiology 1991, 179: 735-8.

4. Ohta H, Nozawa, S. [Postmenopausal osteoporosis. -Mainly on a decline of estrogen and acceleration of the bone resorption-.] Journal of Clinical and Experimental Medicine 1995, 175: 131-5. Japanese.

5. Yamazaki K, Inoue T. Postmenopausal osteoporosis. In Orimo, H. ed. Saishin kotsusoshosho [The lasted osteoporosis]. Life science publication Co., Ltd.: Tokyo, Japan, 1999, pp. 47-51.

6. Banba N. Change by the aging -Internal secretion metabolism-. Dokkyo Journal of Medical Sciences 2008, 35: 209-18.

7. Kanis J.A, Johnell O, Oden A, Sembo I, Redlund-Johnell I, Dawson A, De Laet C, Jonsson B. Long-term risk of osteoporotic fracture in Malmö. Osteoporosis International 2000, 11: 669-74.

8. Howe, T.E, Shea B, Dawson L.J, Downie F, Murray A, Ross C, Harbour R.T, Caldwell L.M, Creed G. Exercise for preventing and treating osteoporosis in postmenopausal women. Cochrane Database Syst Rev 2011, 7:CD000333.

9. Nguyen T.V, Center J.R, Eisman J.A. Osteoporosis: underrated, underdiagnosed and undertreated. Med. J. Aust. 2004, 180: S18-22.

10. Hirota T, Nara M, Ohguri M, Manago E, Hirota K. Effect of diet and lifestyle on bone mass in Asian young women. Am. J. Clin. Nutr. 1992, 55: 1168-73.

11. Yamamura J, Aoe S, Toba Y, Motouri M, Kawakami H, Kumegawa M, Itabashi A, Takada Y. Milk basic protein (MBP) increases radial bone mineral density in healthy adult women. Bioscience Biotechnology, and Biochemistry 2002, 66: 702-4.

12. Aoe S, Toba Y, Yamamura J, Kawakami H, Yahiro M, Kumegawa M, Itabashi A, Takada Y. Controlled trial of the effects of milk basic protein (MBP) supplementation on bone metabolism in healthy adult women. Bioscience Biotechnology, and Biochemistry 2001, 65: 913-8.

13. Aoe S, Koyama T, Toba Y, Itabashi A, Takada Y. A controlled trial of the effect of milk basic protein (MBP) supplementation on bone metabolism in healthy menopausal women. Osteoporosis International 2005, 16: 2123-8.

14. Leib E.S, Lewiecki E.M, Binkley N, Hamdy R.C, International Society for Clinical Densitometry. Official positions of the International Society for Clinical Densitometry. J Clin Densitom 2004, 7: 1-6.

15. Cummings S.R, Black D.M, Nevitt M.C, Browner W, Cauley J, Ensrud K, Genant H.K, Palermo L, Scott J, Vogt T.M. Bone density at various sites for prediction of hip fractures. The Lancet 1993, 341: 72-5.

16. Purwosunu Y, Muharram, Rachman I.A, Reksoprodjo S, Sekizawa A. Vitamin K2 treatment for postmenopausal osteoporosis in Indonesia. J. Obstet. Gynaecol. Res. 2006, 32: 230-4.

17. Jiang Y, Zhang Z.L, Zhang Z.L, Zhu H.M, Wu Y.Y, Cheng Q, Wu F.L, Xing X.P, Liu J.L, Yu W, Meng X.W. Menatetrenone versus alfacalcidol in the treatment of Chinese postmenopausal women with osteoporosis: a multicenter, randomized, double- blinded, double- dummy, positive drug-controlled clinical trial. Clin Interv Aging 2014, 9: 121-7.

18. Ikeda H, lida T, Hiramitsu M, Inoue T, Aoi S, Kanazashi M, Ishizaki F, Harada. T. Effects of lemon beverages on bone metabolism and bone mineral density in postmenopausal women: A double-blind, controlled intervention study with Ca-supplemented and unsupplemented lemon beverages. Open $\mathrm{J}$ Prev Med 2018, 8: 301-14.

19. Parfitt $\mathrm{AM}$, Morphologic basis of bone mineral measurement $囚$ ransient and steady state effects of treatment in osteoporosis $₫$ Miner Electrolyte Metab 1980, 4: 273ه87ه

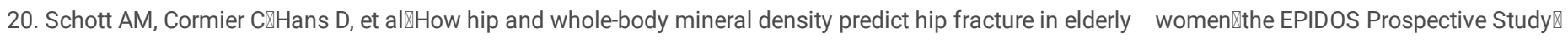
Osteoporos Int 1998, 8: 245-7.

21. Leib ES1, Lewiecki EM, Binkley N, Hamdy RC, International Society for Clinical Densitometry. Official positions of the International Society for Clinical Densitometry. Journal of Clinical Densitometry 2004, 7(1): 1-6.

22. Making committee of the prevention and treatment guidelines on osteoporosis. The prevention and treatment guidelines on osteoporosis 2015 version. Japan Osteoporosis Society, The Japanese Society for Bone and Mineral Research, Japan Osteoporosis Foundation, 2015, Japanese.

23. Albright F, Smith P.H, Richardson A.M. Postmenopausal osteoporosis. JAMA 1941, 116: 2465-74.

24. Gorai I. [Change of Bone Metabolism with Ageing - In Relation to Involvement of Estrogen Withdrawal -]. Acta Obstet Gynaecol Jpn 1997, 49 : 537-45. Japanese.

25. lida T, Ishizaki F, Hayashi M, Ishikawa H, Koyama T, Sawada S, Kishida N. [Effect of menopause on bone metabolism indices and bone mineral density.study of the usefulness of bone metabolism indices-]. Japanese Journal of Clinical Chemistry 2001, 30: 91-9. Japanese.

26. The summary of the Japanese people meal intake standard (2015 version) report [Internet]. Ministry of Health, Labour and Welfare, 2015, Available from: http://www.mhlw.go.jp/file/04-Houdouhappyou-10904750-Kenkoukyoku-Gantaisakukenkouzoushinka/0000041955.pdf. Accessed 25 March 2019.

27. Domoto T, Ishihara K, Miyake Y, Hiramitsu M, Takatera K, Kato Y, Shiraiwa K, Togo M, Takigawa A. Effect of daily lemon intake on a range of parameters related to metabolic syndrome. Health Sciences 2010, 26: 210-8.

28. Smith E.L, Gilligan C, Smith P.E, Sempos C.T. Calcium supplementation and bone loss in middle-aged women. Am. J. Clin. Nutr. 1989, 50: 833-42.

29. Reid I.R, Ames R.W, Evans M.C, Sempos C.T. Long-term effects of calcium supplementation on bone loss and fractures in postmenopausal women: a randomized controlled trial. Am. J. Med. 1995, 98: 331-5.

30. Zou Z.Y, Lin X.M, Xu X.R, Xu R, Ma L, Li Y, Wang M.F. Evaluation of milk basic protein supplementation on bone density and bone metabolism in Chinese young women. Eur J Nutr 2009, 48: 301-6.

31. Orimo H, Shiraki M, Tomita A, Morii H, Fujita T, Ohta M. Effects of menatetrenone on the bone and calcium metabolism in osteoporosis: a double- blind placebo- controlled study. Journal of Bone Mineral Metabolism 1998, 16: 106-12.

32. Shiraki M, Shiraki Y, Aoki C, Miura M. Vitamin K2 (menatetrenone) effectively prevents fractures and sustains lumbar bone mineral density in osteoporosis. Journal of Bone Mineral Research 2000, 15: 515-21. 
33. Ushiroyama T, Ikeda A, Ueki M. Effect of continuous combined therapy with vitamin K2 and vitamin D3 on bone mineral density and coagulofibrinolysis function in postmenopausal women. Maturitas 2002, 41: 211-21.

34. Penniston K.L, Nakada S.Y, Holmes R.P, Assimos D.G. Quantitative assessment of citric acid in lime juice, and commercially - available fruit juice products. J Endourol. 2008, 22: 567-70.

35. Lacour B, Taedivel S, Drüeke T. Stimulation by citric acid of calcium and phosphorous bioavailability in rats fed a calcium-rich diet. Miner Electrolyte Metab 1997, 23: 79-87.

36. Pak C.Y.C, Harvey J.A, HSU M.C. Enhanced calcium bioavailability from a solubilized from of calcium citrate. J. Clin. Endocrinol. Metab. 1987,65 : $801-5$.

37. Miller J.Z, Smith D.L, Flora L, Slemenda C, Jiang X.Y, Johnston C.C. Jr. Calcium absorption from calcium carbonate and a new form of calcium (CCM) in healthy male and female adolescents. Am. J. Clin. Nutr. 1988, 48: 1291-4.

38. Ikeda H, lida T, Hiramitsu M, Takashi I, Okada M, Yoshikawa K, Goto T, Shigematu S, Watanabe M, Kanbara Y, Kita S, Kunimura K, Harada T. The effects of a calcium-fortified lemon drink on bone density and bone metabolism in postmenopausal women. International Medical Journal 2017, $24: 279-83$.

39. Kishikawa Y. Actual situation of ucOC examination in osteoporosis treatment. Orthopedics \& traumatology 2011, 60: 477-9.

40. Villa J.K.D, Diaz M.A.N, Pizziolo V.R, Martino H.S.D. Effect of vitamin K in bone metabolism and vascular calcification: A review of mechanisms of action and evidences. Crit Rev Food Sci Nutr 2017, 57: 3959-70.

41. Liu G, Peacock M. Age-related changes in serum undercarboxylated osteocalcin and its relationships with bone density, bone quality, and hip fracture. Calcif Tissue Int. 1998, 62: 286-9.

42. Nakashima T, Hayashi M, Takayanagi H. New insights into osteoclastogenic signaling mechanism. Trends Endocrinology \& Metabolism 2012, 23: 582-

90 .

43. Suzuki Y. Bone metabolism markers. The Journal of the Japanese Society of Internal Medicine 2007, 96: 2151-8.

44. Riggs B.L. Pathogenesis of osteoporosis. Am.J. Obstet. Gynecol. 1987, 156: 1342-6.

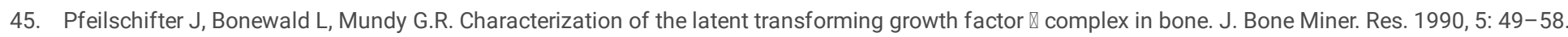

46. Yamamoto K. [The effects of fracture of the femoral neck on the lives of patients and their families]. Acta Scientiarvm Valettvdinis Universitatis

Praefectvralis Ibarakiensis 1996, 1: 55-64. Japanese.

47. Fukunaga M, Sone T. [The measurement of the bone density]. The Journal of the Japanese Society of Internal Medicine 2005, 94: 670-4. Japanese.

48. Public welfare statistics handbook. Mean of Height, the Weight, Sex, Annual× Age distinction [Internet]. Ministry of Health, Labour and Welfare, 2017. Available from: https://www.mhlw.go.jp/toukei/youran/indexyk_2_1.html. Accessed 10 May 2019.

\section{Figures}

(a)

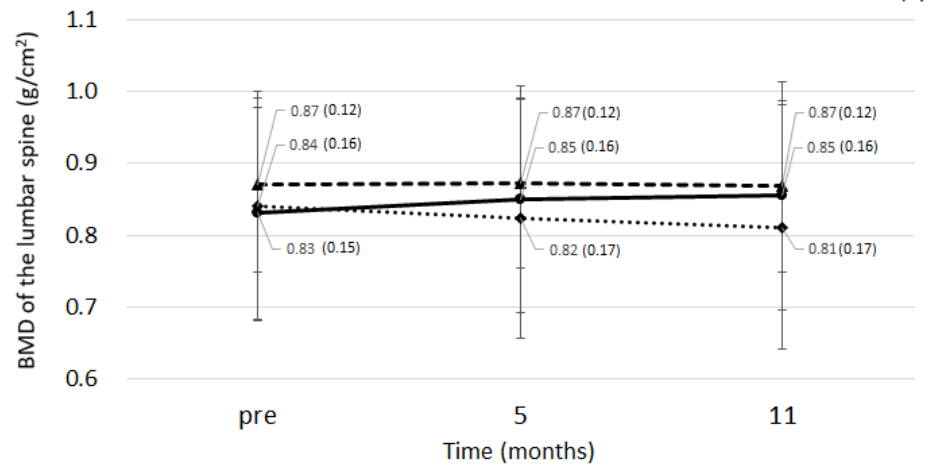

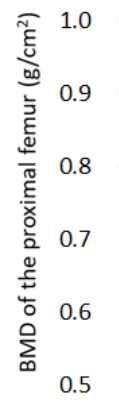

0.5

\section{Figure 1}

Relationship between investigational products and BMD1 (a) BMD of the lumbar spine (b) BMD of the proximal femur Figure shows means and SD2 error bars. The dotted line is the control group, the dashed line is the LE3 group, and the solid line is the LECA4 group. Two-way factorial analysis of variance with the kind of investigational product and intervention period as factors. Each p value for the investigational products (Drink), intervention period (Time), and Interaction is shown. (a) Drink: $p=0.666$; Time: $p=0.956$; Interaction: $p<0.001$ (b) Drink: $p=0.771$; Time: $p=0.413$; Interaction: $p<0.0011$ bone mineral density 2 standard deviation 3 They received an unsupplemented lemon beverage. 4 They received a lemon beverage supplemented with Ca. 

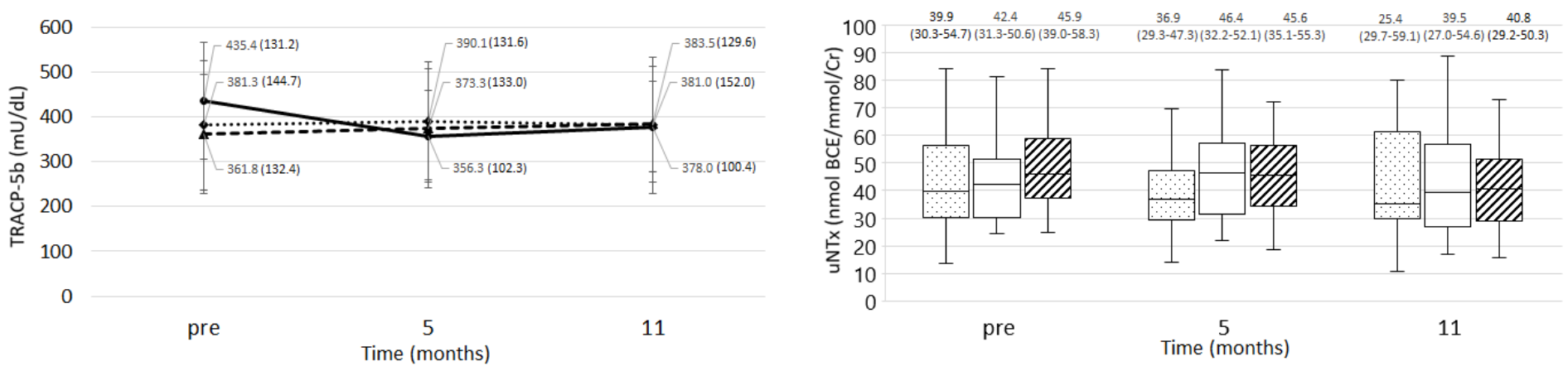

(c)
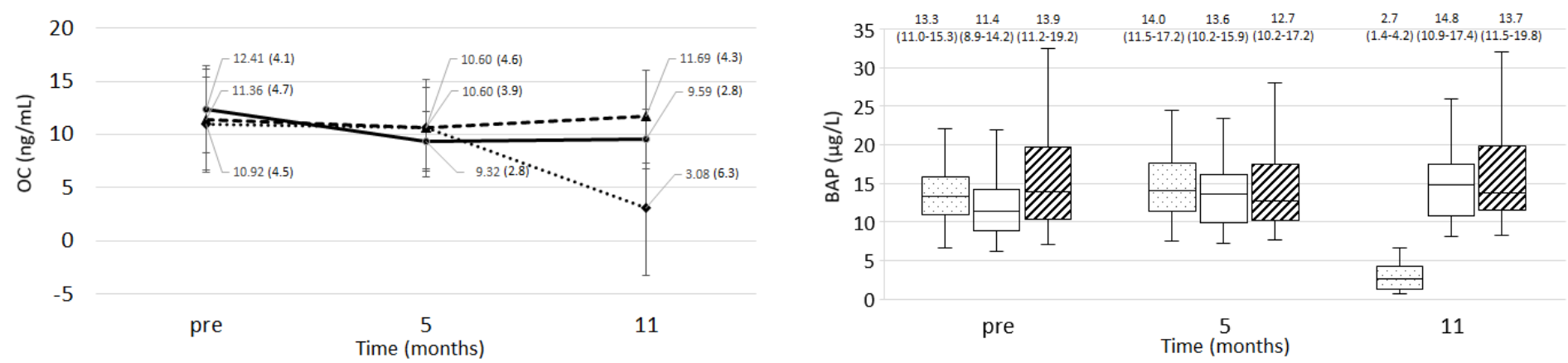

Figure 2

Relationship between investigational products and bone resorption markers (a) Changes in TRACP-5b1 concentrations (b) Changes of each group in uNTx2 (Chronological) (c) Changes in OC3 concentrations (d) Changes of each group in BAP4 concentrations (Chronological) (a, c) Figure shows means and SD5 error bars. The dotted line is the control group, the dashed line is the LE6 group, and the solid line is the LECA7 group. Two-way factorial analysis of variance with the kind of investigational products and intervention period as factors. Each p value for the investigational products (Drink), intervention period (Time), and Interaction is shown ( + ). (a) Drink: $p=0.840$; Time: $p=0.967$; Interaction: $p<0.001+$ (c) Drink: $p=0.788$; Time: $p=0.254$; Interaction: $p=0.006+(b$, $d$ ) Figure shows medians [interquartile range] and SD error bars. The dotted box is the control group, the blank box is the LE group, and the oblique line box is the LECA group. Friedman test with the kind of investigational product and intervention period as factors. The $p$ value of each group is shown ( $¥$ ). (b) Control: $p=0.401 \ddagger$; LE: $p=0.248 \ddagger$; LECA: $p=0.131 \neq$ (d) Control: $p<0.001 \ddagger$; LE: $p<0.001 \neq$; LECA: $p<0.001 \ddagger 1$ tartrate-resistant acid phosphatase type $5 b 2$ urinary type I collagen cross-linked $\mathrm{N}$-telopeptide 3 osteocalcin 4 bone alkaline phosphatase 5 standard deviation 6 They received an unsupplemented lemon beverage. 7 They received a lemon beverage supplemented with $\mathrm{Ca}$.

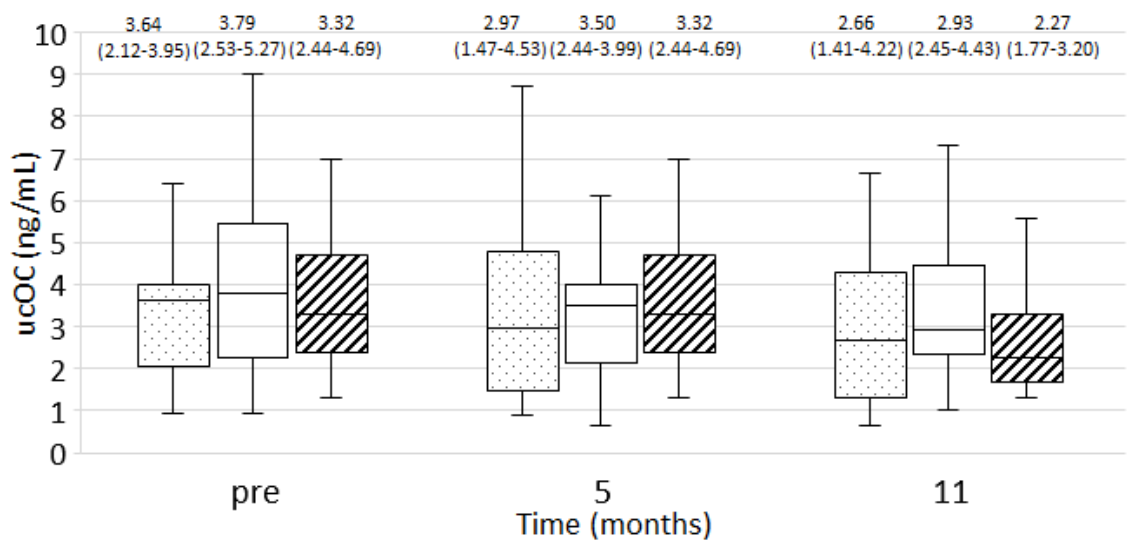

\section{Figure 3}

Changes of each group in ucOC1 concentrations (Chronological) Figure shows medians [interquartile range] and SD2 error bars. The dotted box is the control group, the blank box is the LE3 group, and the oblique line box is the LECA4 group. Friedman test with the kind of investigational product and intervention period as factors. The $p$ value of each group is shown( $\ddagger$ ). Control: $p=0.664 \ddagger ;$ LE: $p=0.214 \ddagger ;$ LECA: $p<0.001 \neq 1$ undercarboxylated osteocalcin 2 standard deviation 3 They received an unsupplemented lemon beverage. 4 They received a lemon beverage supplemented with Ca.

\section{Supplementary Files}


This is a list of supplementary files associated with this preprint. Click to download.

- Flowchart.docx

- CONSORTchecklistikeda.docx

Page $14 / 14$ 